\title{
Virtual Screening Technique Used to Estimate the Mechanism of Adhatoda vasica Nees for the Treatment of Rheumatoid Arthritis Based on Network Pharmacology and Molecular Docking
}

\author{
Wenxiang Wang $\mathbb{D}^{1,2}$ Yunsen Zhang, ${ }^{2}$ Jie Luo, ${ }^{2}$ Rushan Wang, ${ }^{2}$ Ce Tang, ${ }^{3}$ and Yi Zhang $\mathbb{D}^{2}$ \\ ${ }^{1}$ College Pharmacy of Chengdu University of Traditional Chinese Medicine, Chengdu 611137, China \\ ${ }^{2}$ Ethnic Medicine Academic Heritage Innovation Research Center of Chengdu University of Traditional Chinese Medicine, \\ Chengdu 611137, China \\ ${ }^{3}$ Innovative Institute of Chinese Medicine and Pharmacy of Chengdu University of Traditional Chinese Medicine, \\ Chengdu 611137, China \\ Correspondence should be addressed to Yi Zhang; zhangyi_mzyy@163.com
}

Received 20 May 2020; Revised 7 September 2020; Accepted 16 September 2020; Published 29 September 2020

Academic Editor: Monica Borgatti

Copyright (c) 2020 Wenxiang Wang et al. This is an open access article distributed under the Creative Commons Attribution License, which permits unrestricted use, distribution, and reproduction in any medium, provided the original work is properly cited.

\begin{abstract}
Adhatoda vasica Nees (AVN) is commonly used to treat joint diseases such as rheumatoid arthritis (RA) in ethnic minority areas of China, especially in Tibetan and Dai areas, and its molecular mechanisms on RA still remain unclear. Network pharmacology, a novel strategy, utilizes bioinformatics to predict and evaluate drug targets and interactions in disease. Here, network pharmacology was used to investigate the mechanism by which AVN acts in RA. The chemical compositions and functional targets of AVN were retrieved using the systematic pharmacological analysis platform PharmMapper. The targets of RA were queried through the DrugBank database. The protein-protein interaction network (PPI), Gene Ontology (GO), and Kyoto Encyclopedia of Genes and Genomes (KEGG) pathway enrichment analyses of key targets were constructed in the STRING database, and the network visualization analysis was performed in Cytoscape. Maestro 11.1, a type of professional software, was used for verifying prediction and analysis based on network pharmacology. By comparing the predicted target information with the targets of RArelated drugs, 25 potential targets may be related to the treatment of RA, among which MAPK1, TNF, DHODH, IL2, PTGS2, and JAK2 may be the main potential targets for the treatment of RA. Finally, the chemical components and potential target proteins were scored by molecular docking, and compared with the ligands of the protein, the prediction results of network pharmacology were preliminarily verified. The active ingredients and mechanism of AVN against RA were firstly investigated using network pharmacology. Additionally, this research provided a solid foundation for further experimental studies.
\end{abstract}

\section{Introduction}

Rheumatoid arthritis (RA), one of the major diseases leading to disability globally [1], is characterized by joint destruction, pannus formation, synovitis, and adjacent bone erosion [2]. RA can cause cartilage, bone damage, and even disability. RA, a chronic autoimmune disease, is characterized by joint inflammation, and is more like a syndrome that is composed of extra-articular manifestations, such as pulmonary involvement or vasculitis, rheumatoid nodules, and systemic comorbidities [3]. RA affects approximately $1 \%$ of the population, can present at any age, and bring enormous economic and social burden for both the individual and society [4]. Inflammation is the main factor to cause clinical symptoms in RA patients, so anti-inflammation is a key therapeutic strategy [5]. Many drugs such as nonsteroidal compounds, antirheumatic drugs, and glucocorticoids are used to treat RA [6-10]. However, these drugs are associated with many adverse effects and can only slow the progression of RA [11]. With developments in the field of medicine, the treatment of RA has entered a stage of diversified comprehensive treatment. Traditional Chinese medicine (TCM) 
as a supplemental and alternative drug might play a key role in treating RA [12].

Adhatoda vasica Nees (AVN), a popular Ayurvedic medicinal plant in India, is extensively distributed throughout India and tropical regions of South-East Asia including Tibet of China. AVN as an official drug listed in the Indian Pharmacopoeia is commonly used in the preparation of indigenous medicine to treat a variety of diseases such as asthma, cough, bronchitis, and tuberculosis [13]. Moreover, AVN is also commonly used in the treatment of RA in ethnic minority areas of China, especially in Tibetan and Dai areas.

Due to the multichemical components, multipharmacological effects, and multiaction targets of TCM in the treatment of diseases, the traditional research methods are difficult to completely uncover the mechanism of action [14]. However, the network pharmacology produced by the integration of bioinformatics and pharmacology in recent years can distinctly make clear the principle of action of such drugs and can systematically explain the role of multicomponent drugs in the treatment of diseases $[15,16]$. Therefore, we will use the method of network pharmacology to study the effect of AVN in this paper; we used the method of network pharmacology to predict the target of chemical components of AVN, analyzed the interaction between target and metabolic pathway-related RA, and constructed the "component-target-metabolic pathway" network of RA, so as to provide reference for the further study of the material basis and mechanism of anti-RA. It not only provides some information support for the follow-up experimental research but also provides a new way and method for the research of Tibetan medicine.

The workflow of this research on AVN against RA based on network pharmacology is shown in Figure 1.

\section{Materials and Methods}

2.1. Study Design. Chemical constituents of Adhatoda vasica Nees were collected from the public database and published research papers. Then, these compounds were preliminarily screened according to "Lipinski's rule of five." Publicly available databases were utilized for determining human gene/proteins. Genes related to RA were solely selected, which were identified by human disease databases. Pathway analyses related to RA were performed again through publicly available databases. Finally, molecular docking was used to evaluate the interaction of active compounds acting on the targets.

2.2. Chemical Ingredients Database Building. A total of 53 compounds in AVN, including 27 alkaloids, 24 flavonoids, and 2 triterpenoids, were obtained from the previous studies in the public database and published research papers. SMILES formats for the compounds were obtained in the PubChem database [17]. For compounds that were not found in the PubChem database, SMILES formats were generated by ChemDraw (http://www.perkinelmer.com/ category/chemdraw). Their PubChem ID and 2D chemical structures could be obtained on PubChem. Their CAS number could be obtained on the SciFinder database.
2.3. Active Compounds Screening. Traditional medical formulas have hundreds of compounds, but only a minority of them can produce a therapeutic effect. To identify potentially active compounds, we analyze the physicochemical properties of the compounds in the medicinal materials of AVN in this paper. Lipinski's rule of five is a rule of thumb to evaluate druglikeness or determine if a chemical compound with a certain pharmacological or biological activity has chemical properties and physical properties that would make it a likely orally active drug in humans. From the five criteria, molecular weight (MW), hydrogen bond acceptors (Hacc), hydrogen bond donors (Hdon), and octanol-water partition coefficient $\log \mathrm{P}(\mathrm{A} \log \mathrm{P})$ were considered, and hence, we have molecular weight $(\mathrm{MW} \leq 500)$, Moriguchi octanolwater partition coeff $(\log P \leq 5)$, the number of donor atoms of $\mathrm{H}$-bonds $(\mathrm{HD} \leq 5)$, and the number of acceptor atoms for $\mathrm{H}$-bonds $(\mathrm{HA} \leq 10)[18]$.

2.4. Target Prediction. At present, whether active compounds could interact with targets is a critical stage to the drug discovery [19]. Accurate identification and validation of drug-target interactions is the first step on drug discovery pipeline [20]. By PubChem Compound (https://www.ncbi. nlm.nih.gov/pccompound/), we transformed the structure of the candidate compounds into SDF and Canonical SMILES structure format. Swiss Target Prediction (http:// www.swisstargetprediction.ch/) [21] and PharmMapper server databases (http://lilab.ecust.edu.cn/pharmmapper) [22] with the "Homo sapiens" species setting were used for identification of the target genes linked to the selected constituents. The UniProt database (http://www) was utilized for retrieving gene information including name, gene ID, and organism. TTD (http://bidd.nus.edu.sg/BIDDDatabases/TTD/TTD.asp) [23] and DrugBank databases (https://www.drugbank.ca/) were searched for information on RA target genes using only "Homo sapiens" proteins linked to RA. Based on the above methods, 25 distinct targets associated with active constituents and RA were collected.

\subsection{Network Construction}

2.5.1. GO and KEGG Enrichment Analysis of Targets Related to $R A$. To annotate the function of candidate genes and proteins associated with RA, the related biological processes, cellular components, molecular functions, and pathways were analyzed by online STRING 11.0 (https://string-db. org/cgi/help.pl?UserId=PWTj1MTAhQKH and session $\mathrm{Id}=8 \mathrm{wlBKy} 7 \mathrm{kNz} 5 \mathrm{I})$.

2.5.2. Construction of PPI Network for RA Protein Targets. To construct the protein-protein interaction (PPI) network, online STRING 11.0 (https://string-db.org/cgi/help.pl? UserId=PWTj1MTAhQKH\&sessionId=8wlBKy7kNz5I $>$ ) was used to analyze RA-related target proteins [24, 25]. Proteinprotein interactions are critically important to many processes that take place in the cell, including regulation of gene expression, signal transduction, and cell migration. Afterwards, 


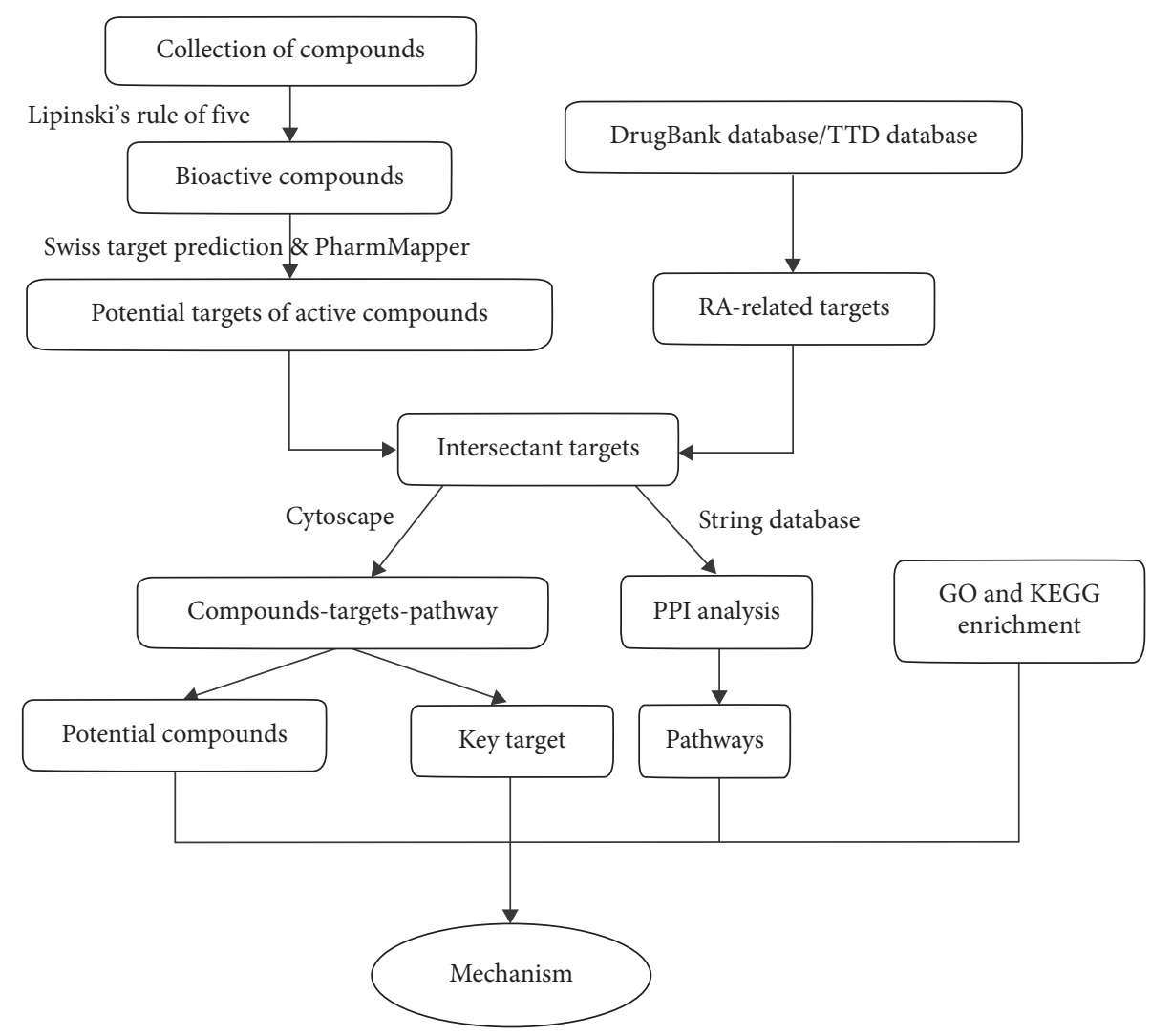

Figure 1: A flow chart of this study.

those RA-related targets were imported into STRING (version 11.0, https://string-db.org/) to investigate protein-protein interactions, and the targets with the species limited to "Homo sapiens" and interaction scores greater than or equal to 0.7 were used as the final targets to conduct GO and KEGG enrichment analysis and network construction.

\subsubsection{Construction of Compound-Target-Pathway Network.} The network of compound-target-pathway was constructed using Cytoscape 3.7.0, an open software platform for network construction, analysis, and visualization, to identify the relationships of target proteins with each compound, the involved pathways, and diseases [26].

2.6. Molecular Docking. The crystal structure of screened targets was obtained from the RCSB PDB database. Maestro 11.1, a type of professional software, was used for verifying prediction and analysis based on network pharmacology and then conducting docking simulation and molecular pathway map. The candidate compounds were downloaded from PubChem and the SDF format using ChemDraw software was generated; candidate target proteins were transformed into PDB ID, and then, they were uploaded to Maestro 11.1 to get docking scores. A conventional rating of docking score $\geq 8.00$ is considered very effective. However, in this paper, we innovatively proposed to use the ligand of protein as contrast, which greatly improved the accuracy of molecular docking results.

\section{Results}

3.1. Chemical Distribution of $A V N$. The effective information of selected compounds is demonstrated in Table S1. According to Lipinski's rule, those compounds whose MW was not more than 500 Daltons, AlogP and Hdon were not more than 5, and Hacc was not more than 10 were thought more likely to be the candidate drugs. However, some compounds, which might not meet this requirement but had significantly pharmacological activities supported by the literature, should also be adopted, such as kaempferol-3-O-rutinoside. The screening process of candidate compounds is shown in Table S2, and at length, 53 ingredients from AVN remained as 42 .

3.2. Target Proteins of AVN. Searching for protein targets of AVN using traditional methods required a lot of manpower, material, and financial resources, and thus, the in silico model was utilized to provide a fast, efficient, and high-throughput approach to acquire the potential protein targets. Based on the pharmacophore matching method, some statistic factors, similarity measures, and so on, 344 targets of $H$. sapiens were obtained, in which PharmMapper contained 142 and Swiss contained 202, and then, the function of each target was obtained from UniProt and the published literature studies (Table S3).

3.3. Identification of RA Target Genes. The results of DrugBank and TTD databases retrieval illustrated that 199 genes were related to RA, and the detailed information is listed in Table S4. 
3.4. GO and KEGG Enrichment Analysis for Targets. Online STRING 11.0 was used to elucidate the biological processes, cell component, and molecular function annotation of the selected 25 targets (Figure 2). There are 611 GO entries (Figure 2 shows the top 10 according to FDR $<0.03$ ), of which 547 entries are related to biological processes, including response to oxygen-containing compounds, regulation of apoptotic process, cellular response to chemical stimulus, negative regulation of apoptotic process, cellular response to oxygen-containing compounds, response to hormones, and response to toxic substances. 52 items are related to molecular functions, including catalytic activity, oxidoreductase activity, enzyme binding, monocarboxylic acid binding, anion binding, cofactor binding, ion binding, signaling receptor binding, and small molecule binding, and 12 cell component entries include membrane raft, focal adhesion, caveola, extrinsic components of the cytoplasmic side of plasma membrane, extracellular regions, side of membrane, neuronal cell body, cytosol, and cytoplasmic part (Table S5). Although a great deal of references manifest that the biological pathways involved in the target proteins of the chemical constituents in AVN are tightly bound to RA, further experimental verification still remains to determine the relationship between active compounds in AVN and their biological pathways.

To further determine the relationship between target proteins and biological pathways, we established a targetpathway network utilizing the online STRING database. 64 pathways corresponding to 25 protein targets were screened (Figure 3 shows the top 15 according to FDR $<0.0001$ ) based on the KEGG analysis with FDR $<0.01$, including pathways in cancer, PPAR signaling pathway, Th17 cell differentiation, toxoplasmosis, PI3K-Akt signaling pathway, and IL-17 signaling pathway. There is one target protein present in many pathways at a time, and several target proteins that exist in one pathway (Table S6).

Generally, one pathway involving many target proteins is more significant than one protein target interacting with many pathways. Hence, we should attempt to seek those pathways related to more target proteins. These results uncover that the active compounds of AVN may achieve the purpose of treating $\mathrm{RA}$ by regulating these signaling pathways.

\subsection{Protein-Protein Interaction Network Construction. A PPI} network (Figure 4) was constructed in order to clarify the relationship between the 25 RA-related targets (Table 1). The nodes in the disease PPI network represent the interrelationships during the development of RA. We constructed a total of 20 nodes and 56 edges in the PPI network of RA. The color of the node is positively related to the degree of contribution of the node in the network. The color of nodes such as MAPK1, TNF, IL2, PTGS2, and JAK2 are darker and can be easily found in the PPI network, and the corresponding degree values (Table 2 ) of each node are 17, 14, 12, 12 , and 10 . These targets may play a key role in the development of RA disease.
From the above results, it is obvious that the five proteins of MAPK1, TNF, IL2, PTGS2, and JAK2 are located at the crucial position, which are mainly involved in MAPK, TNF, JAK, and PI3K-Akt signaling pathways, thus regulating the occurrence and development of RA. Among them, TNF- $\alpha$ has a pleiotropic effect on cell growth, differentiation, cell killing, and inflammation. It also involves the pathogenesis of various autoimmune diseases, such as RA [27], which plays an important role in islet $\beta$-cell destruction. More reported evidence displayed that TNF- $\alpha$ can induce apoptosis in certain cell types [28], whereas TNF- $\alpha$-activated MAPK is required for TNF- $\alpha$-induced apoptosis.

3.6. Compound-Target-Pathway Network Analysis of AVN. To further clarify the potential targets, a component-target-pathway network was established based on the network pharmacology as shown in Figure 5. The network embodies chemical components, target proteins, and metabolic pathways, consisting of 75 nodes and 163 edges. 35 components interact with 25 target proteins and are associated with RA through 15 pathways. Compared with all the target proteins mentioned above, the 25 target proteins in this network are more possibly to be the potential targets of AVN. However, a great quantity of animal experiments and clinical trials are still needed to verify these predictions.

3.7. Molecular Docking. A total of 42 compounds were selected for docking on the 6 target proteins under the procedure as shown in Figure 6, and the molecular docking scores of the compounds are summarized in Table S7. The score of the small molecular ligand docking on the target protein complex was referred to as the threshold, so these compounds docking on this protein with a score greater than this threshold value are considered as the active compounds acting on the target. Finally, we summarize the top three chemical components bound to the key target displayed in Table 3, and the highest scoring compound with key proteins is visualized by molecular docking in Figure 7.

\section{Discussion}

The network pharmacology technique is relatively novel and was proposed by Li et al. for the first time [29]. It provides a deeper understanding of systems biology and network theory, and it has been considered the extra paradigm in drug discovery [30-34]. Network pharmacology has been used to investigate metabolic pathways between drugs and target proteins or genes and diseases, and it can describe complexities among biological systems, drugs, and diseases from informatics perspective [35-37]. Therefore, the development of network pharmacology interacting with molecular docking techniques that can predict multiple drug-target relationship may achieve future drug discoveries in complex diseases such as RA. Here, we integrated various information from publicly available databases to predict the mechanism between AVN and its potential targets related to $\mathrm{RA}$, as well as the signaling pathways 


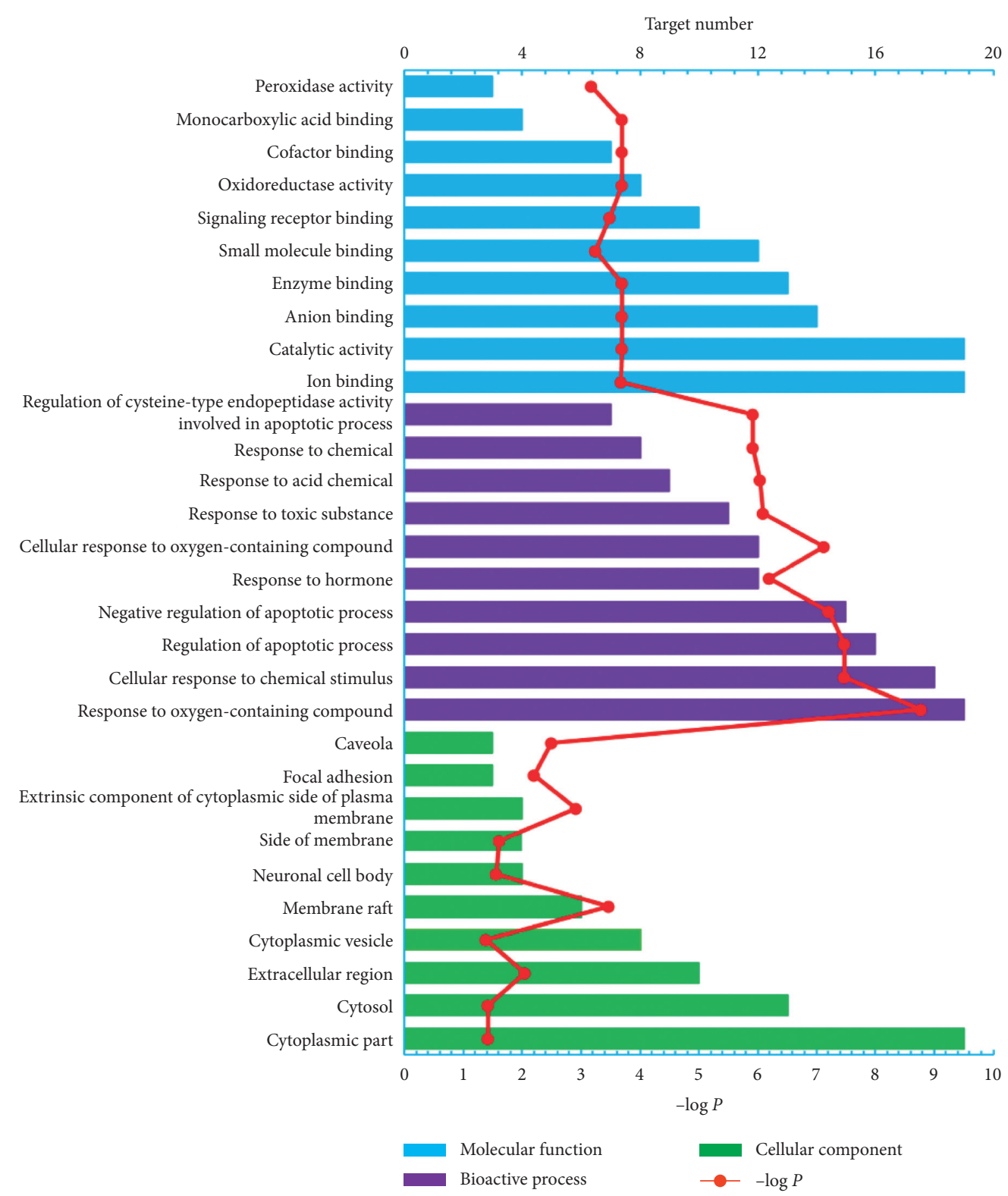

Figure 2: GO enrichment analysis of biological processes, cell composition, and molecular function annotation of the selected 25 target proteins.

involved. Increased mortality in RA is widely recognized [38], so it is very significant to develop safe and effective drugs to antagonize RA. TCM has an irreplaceable effect in treatment of RA in China [39]. However, the active compounds and mechanisms of TCM against RA remain still unclear, and AVN is one of them. In this paper, network pharmacology and molecular docking were employed to tackle these issues, and the results of the present study illuminated the active compounds and mechanisms of AVN against RA based on the holistic perspective and the characteristic of TCM.

The KEGG official website indicated that inflammatory cell infiltration, synovial pannus formation, angiogenesis, bone resorption, and joint destruction are the crucial biological effects involved in RA signaling pathways, and these biological effects were directly related to RA pathogenesis [40-42].

Previous investigations indicated that synovial inflammatory cells were obviously decreased after the anti-TNF- $\alpha$ $\mathrm{mAb}$ treatment, manifesting that TNF- $\alpha$ played an important role in RA pathogenesis [43-45]. The pathogenesis of RA was overexpressed inflammatory cytokines and tissue injury mediated by NF- $\kappa \mathrm{B}$ activation, and drugs could alleviate symptoms of RA by blockade of NF- $\kappa \mathrm{B}$ activation $[46,47]$. Recent work demonstrated that the insufficient apoptosis of inflammatory cells in an RA patient might contribute to pathogenesis and induction of inflammatory cell apoptosis is a feasible strategy for treating RA [48]. These reports confirmed the correctness and rationality of prediction of the molecular mechanisms of AVN against the RA 


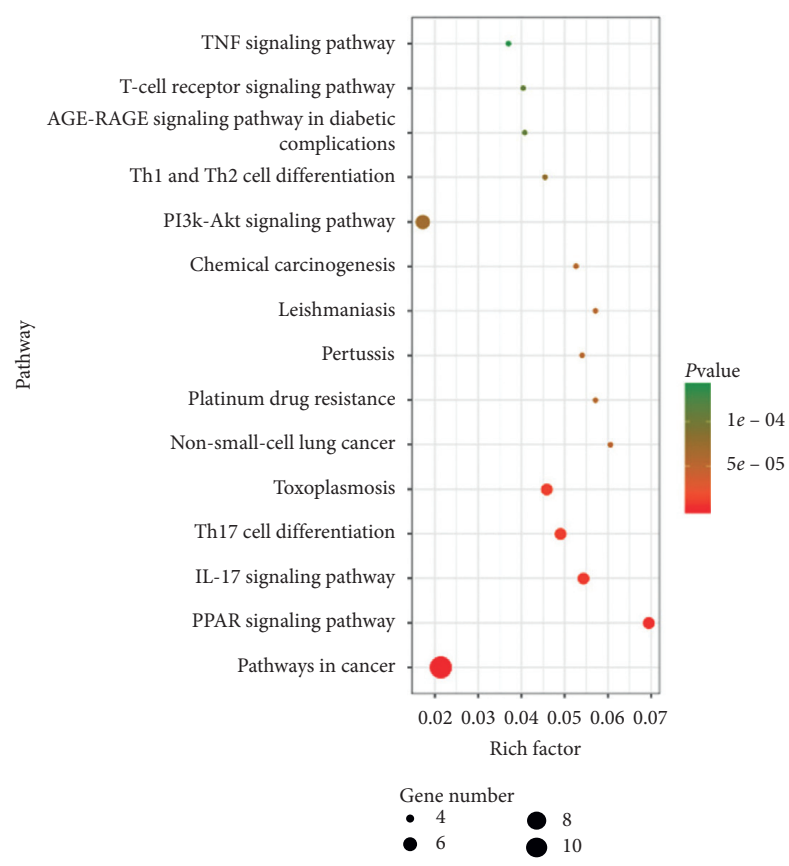

(a)

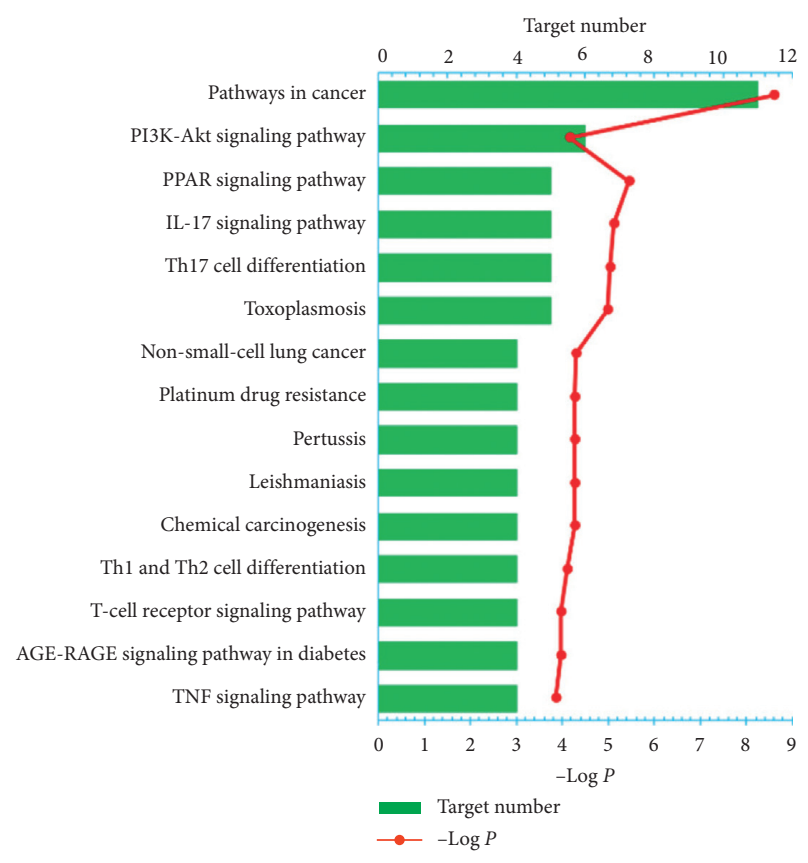

(b)

Figure 3: KEGG analysis of potential targets related to occurrence and development of rheumatoid arthritis: (a) Bubble chart and (b) Histogram.

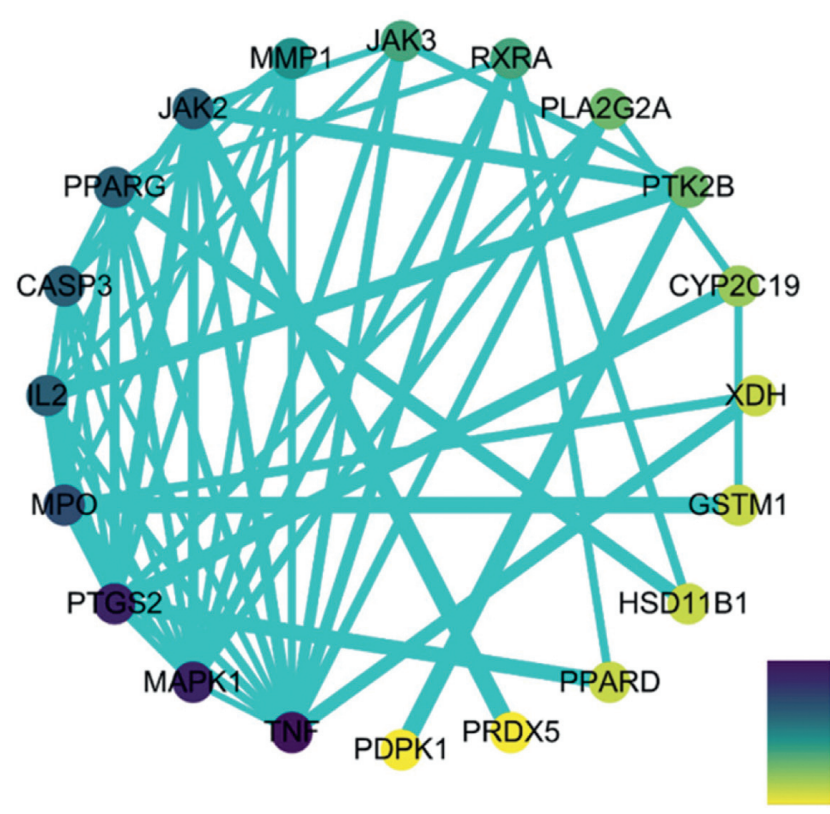

(a)

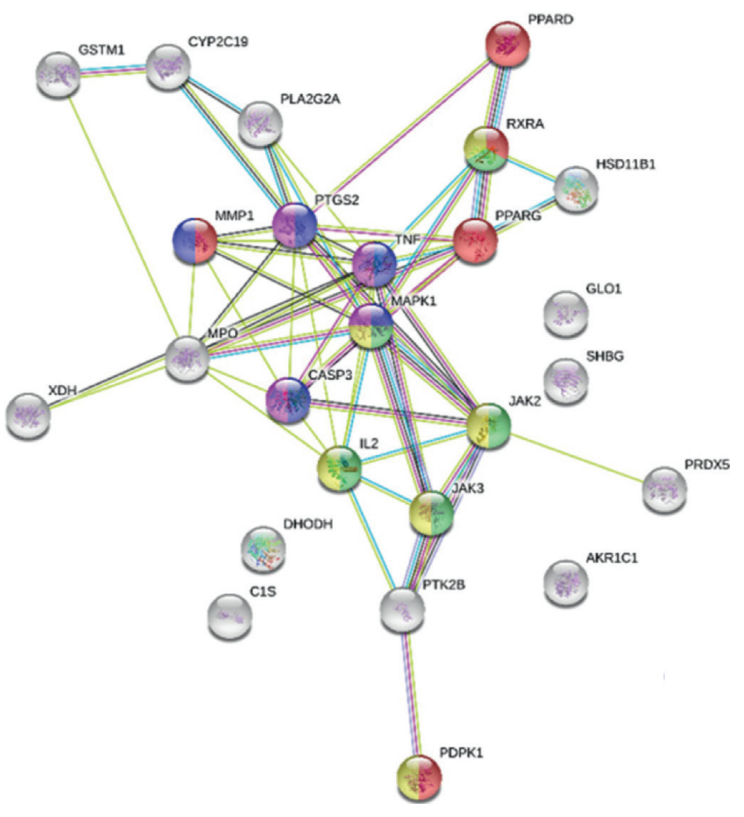

- PPAR signaling pathway - $\mathrm{IL}-17$ signaling pathway - Th17 cell differentiation
- PI3K-Akt signaling pathway - TNF signaling pathway

(b)

Figure 4: Protein-protein interaction network. (a) Constructed by Cytoscape 3.7.0; the color of the node is positively related to the degree of contribution of the node in the network. (b) Constructed by String 11.0; the color of the node is representative to the different signaling pathway as is shown above. 
TABLE 1: RA-related potential target information.

\begin{tabular}{|c|c|c|c|c|}
\hline Number & UniProt name & Target & Gene & Frequency \\
\hline 1 & Q02127 & Dihydroorotate dehydrogenase (quinone), mitochondrial & DHODH & 13 \\
\hline 2 & P47989 & Xanthine dehydrogenase/oxidase & $\mathrm{XDH}$ & 11 \\
\hline 3 & P35354 & Prostaglandin G/H synthase 2 & PTGS2 & 7 \\
\hline 4 & P01375 & Tumor necrosis factor & TNF & 7 \\
\hline 5 & P60568 & Interleukin-2 & IL2 & 7 \\
\hline 6 & P28845 & Corticosteroid 11-beta-dehydrogenase isozyme 1 & HSD11B1 & 5 \\
\hline 7 & P28482 & Mitogen-activated protein kinase 1 & MAPK1 & 5 \\
\hline 8 & $\mathrm{O} 15530$ & 3-Phosphoinositide-dependent protein kinase 1 & PDPK1 & 4 \\
\hline 9 & P37231 & Peroxisome proliferator-activated receptor gamma & PPARG & 4 \\
\hline 10 & P52333 & Tyrosine-protein kinase JAK3 & JAK3 & 4 \\
\hline 11 & Q04760 & Lactoylglutathione lyase & GLO1 & 3 \\
\hline 12 & P19793 & Retinoic acid receptor RXR-alpha & RXRA & 3 \\
\hline 13 & O60674 & Tyrosine-protein kinase JAK2 & JAK2 & 3 \\
\hline 14 & P05164 & Myeloperoxidase & MPO & 2 \\
\hline 15 & Q04828 & Aldo-keto reductase family 1 member $\mathrm{C} 1$ & AKR1C1 & 2 \\
\hline 16 & P30044 & Peroxiredoxin-5, mitochondrial & PRDX5 & 2 \\
\hline 17 & P04278 & Sex hormone-binding globulin & SHBG & 2 \\
\hline 18 & Q03181 & Peroxisome proliferator-activated receptor delta & PPARD & 2 \\
\hline 19 & P42574 & Caspase- 3 & CASP3 & 2 \\
\hline 20 & P33261 & Cytochrome P450 2C19 & CYP2C19 & 1 \\
\hline 21 & Q14289 & Protein-tyrosine kinase 2-beta & PTK2B & 1 \\
\hline 22 & P03956 & Interstitial collagenase & MMP1 & 1 \\
\hline 23 & P09488 & Glutathione S-transferase Mu 1 & GSTM1 & 1 \\
\hline 24 & P14555 & Phospholipase A2, membrane associated & PLA2G2A & 1 \\
\hline 25 & P09871 & Complement $\mathrm{C} 1 \mathrm{~s}$ subcomponent & $\mathrm{C} 1 \mathrm{~S}$ & 1 \\
\hline
\end{tabular}

TABLE 2: The degree value of the target proteins.

\begin{tabular}{|c|c|c|c|c|}
\hline Target & Abbreviation & UniProt ID & PDB ID & Degree \\
\hline Tyrosine-protein kinase JAK2 & JAK2 & O60674 & $4 Z 32$ & 10 \\
\hline Dihydroorotate dehydrogenase (quinone), mitochondrial & $\mathrm{DHODH}$ & Q02127 & 5TCE & 13 \\
\hline Mitogen-activated protein kinase 1 & MAPK1 & P28482 & 4QP3 & 17 \\
\hline Interleukin-2 & IL2 & P60568 & 3QB1 & 12 \\
\hline Tumor necrosis factor & TNF & P01375 & 5 MU8 & 14 \\
\hline Prostaglandin $\mathrm{G} / \mathrm{H}$ synthase 2 & PTGS2 (COX2) & P35354 & $5 \mathrm{~F} 1 \mathrm{~A}$ & 12 \\
\hline
\end{tabular}

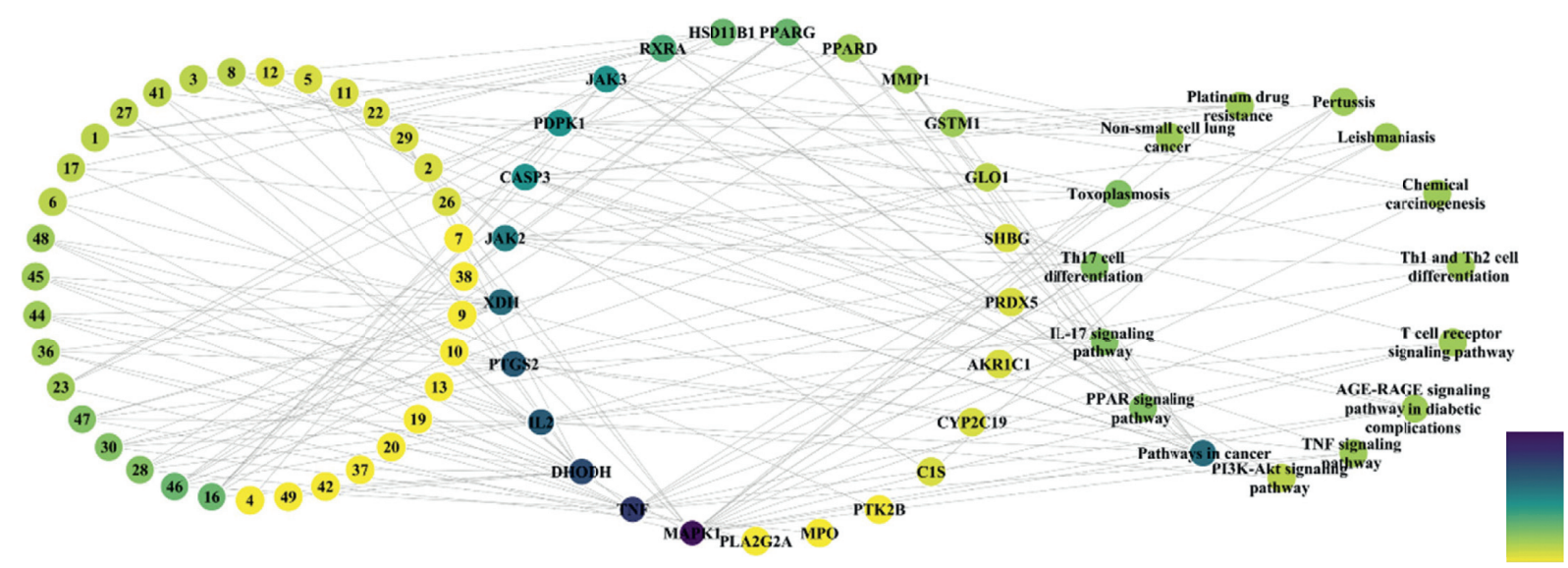

Figure 5: Component-target-pathway network (The color of the node is positively related to the degree of contribution of the node.). 


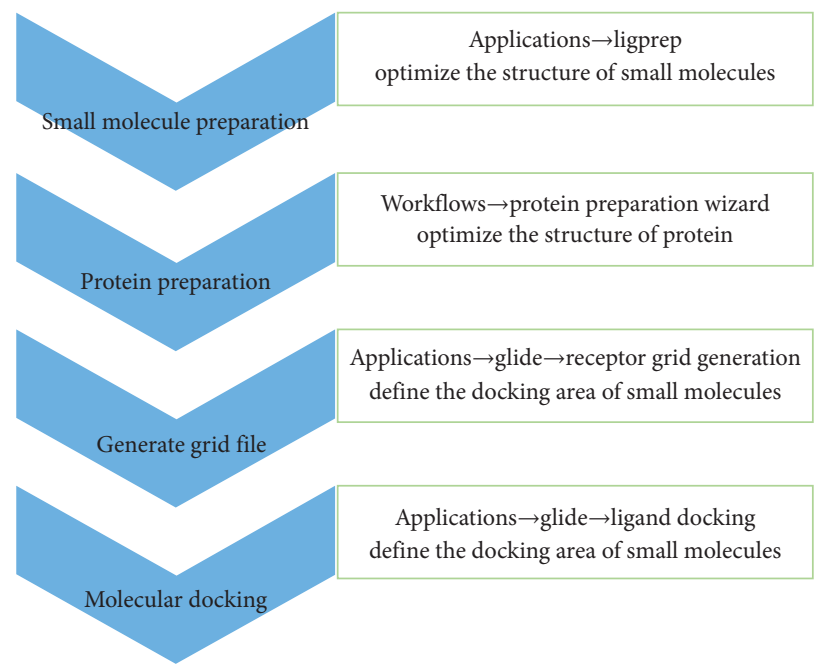

Figure 6: Procedure of molecular docking.

TABLE 3: The docking score of the top three chemical components bound to the key targets.

\begin{tabular}{|c|c|c|c|c|c|c|}
\hline Protein & Chemical constituents & Glide gscore & Glide hbond & Glide evdw & Glide ecoul & Glide energy \\
\hline \multirow{4}{*}{ MAPK1 } & Contrast & -8.969 & -0.32 & -45.135 & -5.983 & -51.118 \\
\hline & Kaempferol-3-O-rutinoside & -9.947 & -0.551 & -56.092 & -25.812 & -81.904 \\
\hline & Quercetin-3-O-rutinoside & -9.385 & -0.315 & -63.010 & -18.544 & -81.554 \\
\hline & Kaempferol-3-O-glucoside & -8.865 & -0.509 & -46.273 & -19.352 & -65.624 \\
\hline \multirow{4}{*}{ TNF } & Contrast & -7.971 & 0 & -45.563 & -1.936 & -47.499 \\
\hline & Quercetin-3-O-sophoroside & -7.968 & -0.32 & -42.107 & -19.831 & -61.938 \\
\hline & Kaempferol-3-O-rutinoside & -7.905 & -0.289 & -43.940 & -15.03 & -58.969 \\
\hline & Deoxyaniflorine & -7.782 & 0 & -37.262 & -1.324 & -38.586 \\
\hline \multirow{4}{*}{ DHODH } & Contrast & -14.062 & -54.687 & -9.844 & -64.531 & -54.687 \\
\hline & N-Demethyl adhatodine & -10.131 & 0 & -51.576 & 0.051 & -51.525 \\
\hline & Adhatodine & -10.077 & 0 & -48.886 & 0.204 & -48.682 \\
\hline & Deoxyaniflorine & -10.01 & -0.535 & -19.431 & -9.260 & -28.691 \\
\hline \multirow{4}{*}{ IL2 } & Contrast & -6.76 & -0.72 & -35.565 & -13.090 & -48.655 \\
\hline & Vasicine & -5.971 & 0 & -22.876 & -1.874 & -24.750 \\
\hline & Vasicine acetate & -5.89 & -0.182 & -23.229 & -4.451 & -27.680 \\
\hline & 1,2,3,9-Tetrahydro-5-methoxypyrrolo-ol & -5.831 & -0.037 & -25.257 & -2.302 & -27.559 \\
\hline \multirow{4}{*}{ PTGS2 } & Contrast & -10.182 & 0 & -41.023 & -10.356 & -51.379 \\
\hline & Quercetin-3-O-rutinoside & -10.301 & 0 & -64.482 & -11.75 & -76.232 \\
\hline & Kaempferol-3-O-sophoroside & -9.721 & -0.154 & -52.359 & -20.364 & -72.723 \\
\hline & Kaempferol-3-O-rutinoside & -9.589 & 0 & -60.938 & -12.074 & -73.013 \\
\hline \multirow{4}{*}{ JAK2 } & Contrast & -10.660 & -0.569 & -41.471 & -7.018 & -48.490 \\
\hline & Quercetin-3-O-sophoroside & -10.520 & -0.174 & -49.761 & -23.978 & -73.739 \\
\hline & Orientin & -9.740 & -0.144 & -31.871 & -27.504 & -59.376 \\
\hline & Quercetin & -9.441 & -0.603 & -32.308 & -18.148 & -50.457 \\
\hline
\end{tabular}

Note: Glide hbond stands for contribution of hydrogen-bonding. Glide evdw stands for van der Waals energy. Glide ecoul stands for Coulomb energy. All items are involved in the Glide gscore algorithm.

network based on pharmacology. The relationships between other signaling pathways and RA were not discussed in detail in this work, but their relationships could be easily identified by retrieving literature data.

The degree value of genes indicated their contribution to the therapeutic effect of AVN on RA, and that PTGS2 was the core gene of AVN against RA. In the 64 signaling pathways, PTGS2 was enriched in TNF and NF- $\kappa \mathrm{B}$ signaling pathways, and the KEGG official website uncovers the effect of PTGS2 in the two signaling pathways by regulating inflammation response. COX-2 enzyme, generated by PTGS2 gene, was induced by proinflammatory cytokines to promote inflammation progression, and its expression was selectively blocked by dexamethasone, a potent anti-inflammatory drug [49]. Inflammation is the crucial factor to trigger RA symptoms, such as joint damage, disability, and comorbidity; hence anti-inflammation is the main therapeutic strategy [5]. The key targets in the compound-target network 


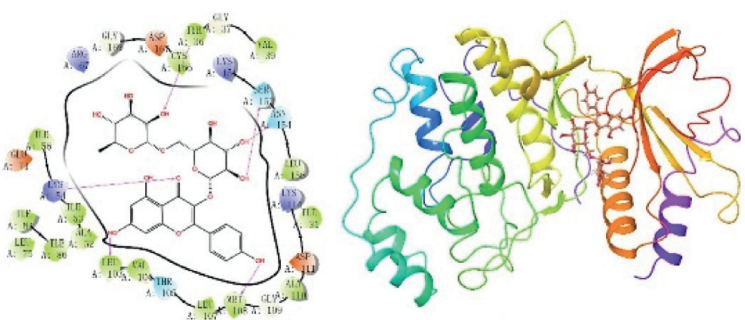

(a)
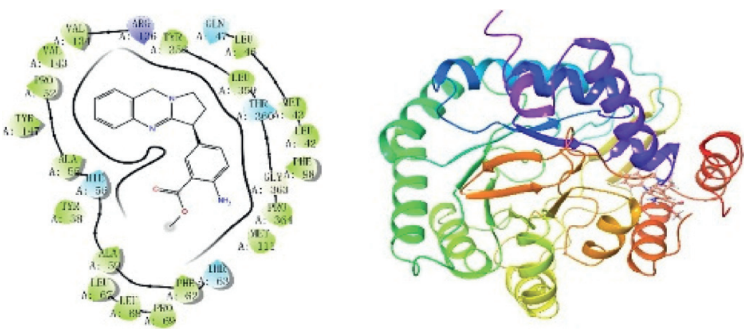

(c)
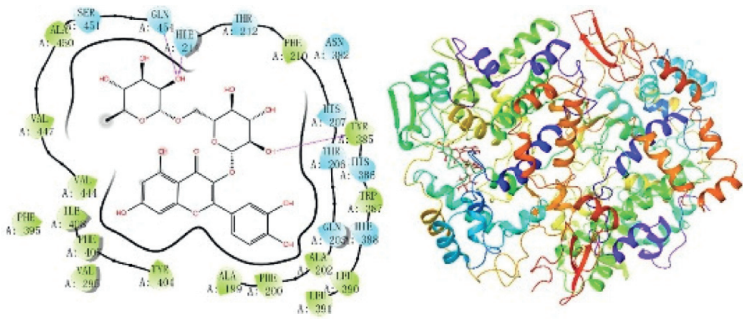

(e)

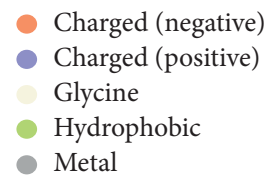

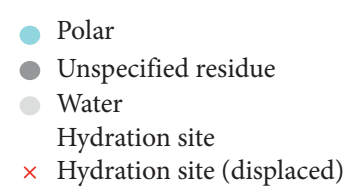

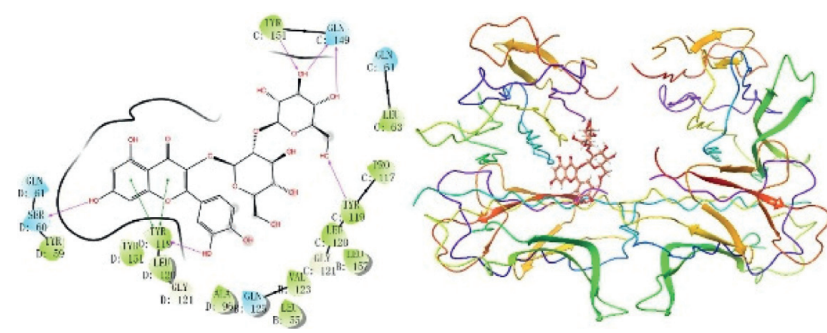

(b)

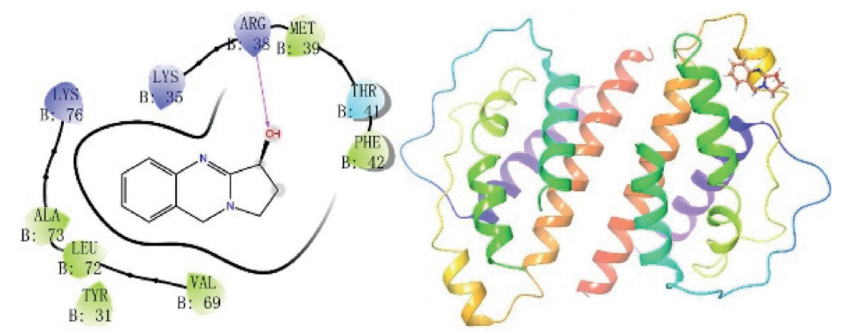

(d)
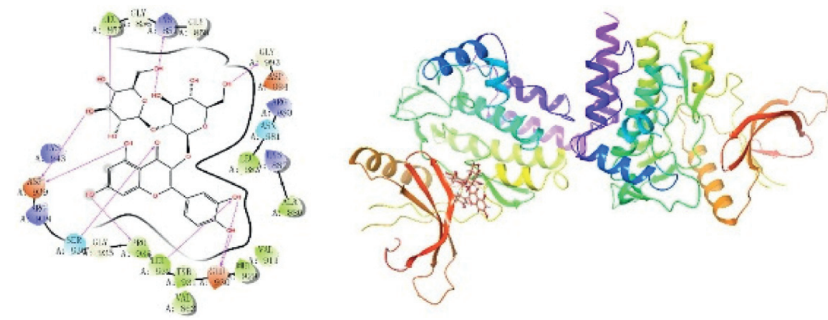

(f)

$$
\begin{aligned}
& -- \text { Distance } \\
& \longrightarrow \text { H-bond } \\
& \longrightarrow \text { Metal coordination } \\
& \longrightarrow \text { Pi-Pi stacking } \\
& \longrightarrow \text { Pi-cation }
\end{aligned}
$$

Figure 7: Molecular docking of active compounds and key targets: (a) kaempferol-3-O-rutinoside to MAPK1; (b) quercetin-3-Osophoroside to TNF; (c) N-demethyl adhatodine to DHODH; (d) vasicine to IL2; (e) quercetin-3-O-rutinoside to PTGS2; and (f) quercetin3-O-sophoroside to JAK2.

of AVN are PTGS2 and MAPK1. These targets are involved in various aspects of disease regulation such as inflammation, control, and endocrine therapy of tumors. PTGS is a key rate-limiting enzyme involved in the synthesis of prostaglandins from arachidonic acid. There are two isomerases: PTGS1 and PTGS2. PTGS2 plays an important role in the development of RA [50]. Studies have shown that the expression of cyclooxygenase is significantly increased in the synovial tissue of patients, accompanied by the induction of prostaglandin E2, and inflammatory factors are synthesized in large quantities. This causes the infiltration of inflammatory cells, abnormal proliferation of synovial tissue, and formation of neovascularization, resulting in swelling and degeneration of joints [51]. Therefore, by inhibiting the expression of PTGS2 in synovial cells, the synthesis of prostaglandin $\mathrm{E} 2$ can be reduced, consequently reducing the inflammatory response of RA and improving the disease [52]. Therefore, AVN or the combination of the above components for some key targets plays an important role in the regulation of inflammatory response. TNF- $\alpha$ can effectively reduce the arthritis and synovitis symptoms of RA patients, and RA can be suppressed by inhibiting the expression of TNF- $\alpha$ [43].

It was reported that andrographolide demonstrated protective effects on RA through regulating MAPK pathways, suggesting that the MAPK signaling pathway was related to occurrence and development of RA [53]. The PI3K-Akt signaling pathway might be the hub signaling pathway of AVN against RA. Joint synovium is the main diseased region in RA patients. Therefore, inducing apoptosis of synovial cells is a feasible strategy for treating RA by preventing development of inflammation [54]. The PI3KAkt signaling pathway was abnormally activated in RA synovium, resulting in the overexpression of antiapoptotic genes such as FLIP, Bcl-2, and Mcl-1 [55]. The overexpression of these antiapoptotic genes results in out-ofbalance apoptosis of synovial cells, which induced RA [56]. Additionally, luteolin, an uppermost active ingredient against RA, inhibited the proliferation of synovial fibroblasts in RA by blocking the PI3K-Akt signaling pathway [57]. 
PPARs (peroxisome proliferation-activated receptors) are ligand-activated transcription factors. PPAR- $\gamma$, a subtype of PPARs, is more closely related to RA. The expression of PPAR- $\gamma$ can be detected in synovial cells involved in RA. PPAR- $\gamma$ agonists can inhibit the hyperplasia of synovial cells and induce their apoptosis [58, 59]. In addition, PPAR- $\gamma$ agonists can inhibit the generation of key mediators in RA from macrophages, including IL- $1 \beta$, IL-6, and TNF- $\alpha$. In conclusion, the PPAR signaling pathway is important in treating RA by intervening with the pathological process of RA through the corresponding receptor agonists. Based on these existing reports, it is reasonable to conclude that the mechanism of AVN against RA was inflammation response through inactivating TNF, MAPK, PPAR, and PI3K-Akt signaling pathways.

The pathophysiological mechanism of RA is specially complicated, and various biological processes and metabolic pathways are involved in the process of RA damage [60]. 198 RA targets screened in this study mainly partake in the release of inflammatory cytokines and proinflammatory factors by TNF, MAPK, and PI3K-AKT signaling pathways [61, 62]. According to the network of "active compound-target-pathway," AVN may intervene the inflammation pathways to reduce the release of proinflammatory factors and inflammatory cytokines through "multicomponent-single target" or "multicomponentmultitarget". Collectively, AVN may have significant potential to treat RA by a combination of multicomponents, multitargets, and multipathways.

As TCM is characterized by multicomponent, multitarget, multilevel, and network-based therapeutic effects, traditional pharmacy, pharmacology, and molecular biology research are not enough to explain its complex mechanism and material basis. Based on the big data platform of network pharmacology research (a database involving multiple drug components, disease targets, protein interactions, and signal pathways), the overall analysis of the material basis and mechanism of action is not only comprehensive but also conducive for refining its main therapeutic efficacy-related targets, components, pathways, and a comprehensive view of the problem. However, there were some limitations to this study. For example, although there are some clinical studies on the anti-inflammatory and immunomodulatory effects of the compounds analyzed, these mechanisms need to be further verified. Although network pharmacology is a simple and efficient method for predicting drug targets in sophisticated diseases, it is still necessary to verify the scientific nature and rationality of predicted targets by in vitro experiments.

\section{Conclusion}

This paper firstly explored the active compounds and molecular mechanisms of AVN against RA based on network pharmacology and molecular docking. The active chemical constituents of AVN against RA consisted of 35 compounds, and peganidine, quercetin-3-O-rutinoside, and quercetin were the key active ingredients. The related genes of AVN against RA included 25 target genes, and MAPK1 and PTGS2 were the hub genes. The mechanism of AVN against RA mainly comprised 15 signaling pathways, and the key mechanism was related to inhibition of inflammation response through inactivating TNF and PPAR signaling pathways. Additionally, this research provided scientific evidence and a good theoretical foundation to support the therapeutic effect of AVN on RA. Although we predicted the possible target proteins by AVN and further verified them by molecular docking, the two methods are prediction after all. Then, we prepare to use effective fraction or active compounds to treat synovial cells in human arthritis, analyzing expression levels on proteins and mRNA levels by transcriptomics and proteomics studies. At length, our results predict that the therapeutic effects of AVN against RA are mediated via MAPK1, TNF, IL2, PTGS2, DHODH, and JAK2. These results may be useful in guiding further research to clearly clarify the molecular targets of AVN related to RA and applications of network pharmacology in drug discovery.

\section{Data Availability}

The data used to support the findings of this study are included within Supplementary Materials.

\section{Conflicts of Interest}

The authors declare that there are no conflicts of interest regarding the publication of this paper.

\section{Authors' Contributions}

Yi Zhang proposed the idea and designed the study. Wenxiang Wang performed the study and wrote the manuscript. Yunsen Zhang and Ce Tang participated in data analysis. Jiu Luo and Rushan Wang improved the manuscript. All authors read and approved the final manuscript.

\section{Acknowledgments}

This study was supported by National Key Research and Development Program of China (no. 2017YFC1703904), National Natural Science Foundation (nos. 81973573, 81973569, and 81903922), Project First-Class Disciplines Development of Chengdu University of Traditional Chinese Medicine (CZYJC1903), and Xinglin Scholar Research Premotion Project of Chengdu University of TCM (CXTD2018015 and CXTD2018020).

\section{Supplementary Materials}

Table S1: effective information of selected compounds. Table S2: screening of candidate compounds. Table S3: a list of genes linked to all candidate compounds from Adhatoda vasica Nees. Table S4: a list of 199 genes linked to RA from DrugBank and TTD database. Table S5: the biological processes, cell composition, and molecular function annotation by String database of the selected 25 target proteins. Table S6: the KEGG analysis by String database of the selected 25 target proteins. Table S7: the docking score of all chemical constituents and MAPK1, IL2, TNF, PTGS2, DHODH, and JAK2. (Supplementary Materials) 


\section{References}

[1] T. Vos, A. A. Abajobir, K. H. Abate et al., "Global, regional, and national incidence, prevalence, and years lived with disability for 328 diseases and injuries for 195 countries," The Lancet, vol. 390, no. 10100, pp. 1211-1259, 2017.

[2] A. K. Rana, Y. Li, Q. Dang, and F. Yang, "Monocytes in rheumatoid arthritis: circulating precursors of macrophages and osteoclasts and, their heterogeneity and plasticity role in RA pathogenesis," International Immunopharmacology, vol. 65, pp. 348-359, 2018.

[3] J. S. Smolen, D. Aletaha, and I. B. McInnes, "Rheumatoid arthritis," The Lancet, vol. 388, no. 10055, p. 2023, 2016.

[4] I. B. McInnes and G. Schett, "Pathogenetic insights from the treatment of rheumatoid arthritis," The Lancet, vol. 389, no. 10086, pp. 2328-2337, 2017.

[5] J. S. Smolen, D. Aletaha, M. Koeller, M. H. Weisman, and P. Emery, "New therapies for treatment of rheumatoid arthritis," The Lancet, vol. 370, no. 9602, pp. 1861-1874, 2007.

[6] P. Conigliaro, P. Triggianese, E. Fonti et al., "Challenges in the treatment of rheumatoid arthritis," Autoimmunity Reviews, vol. 18, no. 7, pp. 706-713, 2019.

[7] J. Alam, I. Jantan, and S. N. A. Bukhari, "Rheumatoid arthritis: recent advances on its etiology, role of cytokines and pharmacotherapy," Biomedicine \& Pharmacotherapy, vol. 92, pp. 615-633, 2017.

[8] G. R. Burmester and J. E. Pope, "Novel treatment strategies in rheumatoid arthritis," The Lancet, vol. 389, no. 10086, pp. 2338-2348, 2017.

[9] I. Cecchi, I. Arias de la Rosa, E. Menegatti et al., "Neutrophils: novel key players in rheumatoid arthritis current and future therapeutic targets," Autoimmunity Reviews, vol. 17, no. 11, pp. 1138-1149, 2018.

[10] T. T. Cheung and I. B. McInnes, "Future therapeutic targets in rheumatoid arthritis?" Seminars in Immunopathology, vol. 39, no. 4, pp. 487-500, 2017.

[11] Y. M. Di, Z. W. Zhou, L. C. Guang et al., "Current and future therapeutic targets of rheumatoid arthritis," Anti-Inflammatory \& Anti-allergy Agents in Medicinal Chemistry, vol. 10, no. 2, pp. 92-120, 2011.

[12] L. L. Li, X. B. Sun, and D. X. Xiang, "Research progress on anti-rheumatic arthritis of active ingredients in natural medicine," Central South Pharmacy, vol. 9, no. 2, pp. 130-134, 2011.

[13] A. Singh, S. Kumar, T. Jagadeshwar Reddy et al., "Screening of tricyclic quinazoline alkaloids in the alkaloidal fraction of Adhatoda beddomei and Adhatoda vasica leaves by highperformance liquid chromatography/electrospray ionization quadrupole time-of-flight tandem mass spectrometry," Rapid Communications in Mass Spectrometry, vol. 29, no. 6, pp. 485-496, 2015.

[14] A. Kumar, D. Sharma, M. L. Aggarwal, K. M. Chacko, and T. K. Bhatt, "Cancer/testis antigens as molecular drug targets using network pharmacology," Tumor Biology, vol. 37, no. 12, pp. 15697-15705, 2016.

[15] L. Chen, D. Lv, D. Wang et al., "A novel strategy of profiling the mechanism of herbal medicines by combining network pharmacology with plasma concentration determination and affinity constant measurement," Molecular BioSystems, vol. 12, no. 11, pp. 3347-3356, 2016.

[16] S. Li, T. P. Fan, W. Jia et al., "Network Pharmacology in Traditional Chinese Medicine," Evidence-Based Complementary and Alternative Medicine, vol. 2014, Article ID 138460, 2014.
[17] Y. Wang, J. Xiao, T. O. Suzek et al., "PubChem: a public information system for analyzing bioactivities of small molecules," Nucleic Acids Research, vol. 37, no. 2, pp. 623-633, 2009.

[18] C. A. Lipinski, F. Lombardo, B. W. Dominy, and P. J. Feeney, "Experimental and computational approaches to estimate solubility and permeability in drug discovery and development settings," Advanced Drug Delivery Reviews, vol. 64, no. 1, pp. 4-17, 2012.

[19] S. Núñez, J. Venhorst, and C. G. Kruse, "Target-drug interactions: first principles and their application to drug discovery," Drug Discovery Today, vol. 17, no. 1-2, pp. 10-22, 2012.

[20] X. Chen, C. C. Yan, X. Zhang et al., "Drug-target interaction prediction: databases, web servers and computational models," Briefings in Bioinformatics, vol. 17, no. 4, pp. 696-712, 2016.

[21] D. Gfeller, A. Grosdidier, M. Wirth et al., "Swiss Target Prediction: a web server for target prediction of bioactive small molecule," Nucleic Acids Research, vol. 42, no. 1, pp. 32-38, 2014.

[22] N. Wang, G. Zhao, Y. Zhang et al., "A network pharmacology approach to determine the active components and potential targets of Curculigo orchioides in the treatment of osteoporosis," Medical Science Monitor, vol. 23, pp. 5113-5122, 2017.

[23] Y. H. Li, C. Y. Yu, X. X. Li et al., "Therapeutic target database update 2018: enriched resource for facilitating bench-to-clinic research of targeted therapeutics," Nucleic Acids Research, vol. 46, no. 1, pp. 1121-1127, 2018.

[24] S. K. Kushwaha and M. Shakya, "Protein interaction network analysis-Approach for potential drug target identification in Mycobacterium tuberculosis," Journal of Theoretical Biology, vol. 262, no. 2, pp. 284-294, 2010.

[25] S. Lee, K. Park, and D. Kim, "Building a drug-target network and its applications," Expert Opinion on Drug Discovery, vol. 4, no. 11, pp. 1177-1189, 2009.

[26] P. Shannon, A. Markiel, O. Ozier et al., "Cytoscape: a software environment for integrated models of biomolecular interaction networks," Genome Research, vol. 13, no. 11, pp. 2498-2504, 2003.

[27] E. E. Powell, L. S. Wicker, L. B. Peterson, and J. A. Todd, "Allelic variation of the type 2 tumor necrosis factor receptor gene,” Mammalian Genome, vol. 5, no. 11, pp. 726-727, 1994.

[28] H. Iwahashi, T. Hanafusa, Y. Eguchi et al., "Cytokine-induced apoptotic cell death in a mouse pancreatic beta-cell line: inhibition by Bcl-2," Diabetologia, vol. 39, no. 5, pp. 530-536, 1996.

[29] X.-J. Li, Z.-Z. Jiang, and L.-Y. Zhang, “Triptolide: progress on research in pharmacodynamics and toxicology," Journal of Ethnopharmacology, vol. 155, no. 1, pp. 67-79, 2014.

[30] R. Al Mahmud, R. A. Najnin, and A. H. Polash, "A survey of web-based chemogenomic data resources," Methods in Molecular Biology, vol. 1825, pp. 3-62, 2018.

[31] A. Huang, G. Fang, Y. Pang et al., "A network pharmacology approach to explore mechanism of action of Longzuan Tongbi formula on rheumatoid arthritis," Evidence-Based Complementary and Alternative Medicine, vol. 2019, p. 13, Article ID 5191362, 2019.

[32] P. Li, J. Chen, J. Wang et al., "Systems pharmacology strategies for drug discovery and combination with applications to cardiovascular diseases," Journal of Ethnopharmacology, vol. 151, no. 1, pp. 93-107, 2014.

[33] S. Li and B. Zhang, "Traditional Chinese medicine network pharmacology: theory, methodology and application," 
Chinese Journal of Natural Medicines, vol. 11, no. 2, pp. 110-120, 2013.

[34] K. Ning, X. Zhao, A. Poetsch et al., "Computational molecular net-works and network pharmacology," BioMed Research International, vol. 2017, Article ID 7573904, 2017.

[35] B. Zhang, X. Wang, and S. Li, "An integrative platform of TCM network pharmacology and its application on a Herbal Formula, Qing-Luo-Yin," Evidence-Based Complementary and Alternative Medicine, vol. 2013, Article ID 456747, 2013.

[36] C. Zheng, J. Wang, J. Liu, M. Pei, C. Huang, and Y. Wang, "System-level multi-target drug discovery from natural products with applications to cardiovascular diseases," Molecular Diversity, vol. 18, no. 3, pp. 621-635, 2014.

[37] W. Zhou, Y. Wang, A. Lu et al., "Systems pharmacology in small molecular drug discovery," International Journal of Molecular Sciences, vol. 17, no. 2, 2016.

[38] E. Myasoedova, J. M. Davis III., C. S. Crowson, and S. E. Gabriel, "Epidemiology of rheumatoid arthritis: rheumatoid arthritis and mortality," Current Rheumatology Reports, vol. 12, no. 5, pp. 379-385, 2010.

[39] P. Zhang, J. Li, Y. Han, X. Wei Yu, and L. Qin, "Traditional Chinese medicine in the treatment of rheumatoid arthritis: a general review," Rheumatology International, vol. 30, no. 6, pp. 713-718, 2010.

[40] P. E. Brenchley, "Antagonising angiogenesis in rheumatoid arthritis," Annals of the Rheumatic Diseases, vol. 60, no. 3, pp. 71-74, 2001.

[41] K. Wang, D. Zhang, Y. Liu et al., "Traditional Chinese medicine formula Bi-Qi capsule alleviates rheumatoid arthritis-induced inflammation, synovial hyperplasia, and cartilage destruction in rats," Arthritis Research \& Therapy, vol. 20, no. 1, p. 43, 2018.

[42] S. Hua, T. H. Dias, D. G. Pepperall et al., "Topical loperamideencapsulated liposomal gel increases the severity of inflammation and accelerates disease progression in the adjuvantinduced model of experimental rheumatoid arthritis," Frontiers in Pharmacology, vol. 8, p. 503, 2017.

[43] H. Matsuno, K. Yudoh, R. Katayama et al., "The role of TNF- $\alpha$ in the pathogenesis of inflammation and joint destruction in rheumatoid arthritis (RA): a study using a human RA/SCID mouse chimera," Rheumatology, vol. 41, no. 3, pp. 329-337, 2002.

[44] S. Bek, A. B. Bojesen, J. V. Nielsen et al., "Systematic review and meta-analysis: pharmacogenetics of anti-TNF treatment response in rheumatoid arthritis," The Pharmacogenomics Journal, vol. 17, no. 5, pp. 403-411, 2017.

[45] M. Feldmann, "Development of anti-TNF therapy for rheumatoid arthritis," Nature Reviews Immunology, vol. 2, no. 5, pp. 364-371, 2002.

[46] N. Mu, J. Gu, T. Huang et al., "A novel NF- $\kappa$ B/YY1/microRNA-10a regulatory circuit in fibroblast-like synoviocytes regulates inflammation in rheumatoid arthritis," Scientific Reports, vol. 6, Article ID 20059, 2016.

[47] Y. Zhuang, J. Liu, P. Ma et al., "Tamarixinin A alleviates joint destruction of rheumatoid arthritis by blockade of MAPK and NF- $\kappa$ B activation," Frontiers in Pharmacology, vol. 8, p. 538, 2017.

[48] R. M. Pope, "Apoptosis as a therapeutic tool in rheumatoid arthritis," Nature Reviews Immunology, vol. 2, no. 7, pp. 527-535, 2002.

[49] K. Seibert and J. L. Masferrer, "Role of inducible cyclooxygenase (COX-2) in inflammation," Receptor, vol. 4, no. 1, pp. 17-23, 1994.
[50] W. X. Jing, "Correlation between vascular endothelial growth factor and rheumatoid arthritis," Modern Chinese Clinical Medicine, vol. 21, no. 1, pp. 15-18, 2014.

[51] H. P. Yao, M. W. Li, L. H. Zhang et al., "Expression of synovial fibroblast cyclooxygenase-2 and inducible nitric oxide synthase in rheumatoid arthritis," Chinese Journal of Laboratory Medicine, vol. 25, no. 6, pp. 345-348, 2002.

[52] Y. H. Zhang, "Effects of shikonin on the expression of COX-2 in synovial fibroblasts of rheumatoid arthritis," Journal of Harbin Medical University, vol. 46, no. 3, pp. 261-265, 2012.

[53] Z.-z. Li, J.-p. Tan, L.-l. Wang, and Q.-h. Li, "Andrographolide benefits rheumatoid arthritis via inhibiting MAPK pathways," Inflammation, vol. 40, no. 5, pp. 1599-1605, 2017.

[54] S. Y. Park, S. W. Lee, H. K. Shin et al., "Cilostazol enhances apoptosis of synovial cells from rheumatoid arthritis patients with inhibition of cytokine formation via Nrf2-linked heme oxygenase 1 induction," Arthritis \& Rheumatology, vol. 62, no. 3, pp. 732-741, 2010.

[55] S. J. Harris, J. G. Foster, and S. G. Ward, "PI3K isoforms as drug targets in inflammatory diseases: lessons from pharmacological and genetic strategies," Current Opinion in Investigational Drugs (London, England: 2000), vol. 10, no. 11, pp. 1151-1162, 2009.

[56] M. D. Smith, H. Weedon, V. Papangelis et al., "Apoptosis in the rheumatoid arthritis synovial membrane: modulation by disease-modifying anti-rheumatic drug treatment," Rheumatology, vol. 49 , no. 5, pp. 862-875, 2010.

[57] Y. Hou, J. Wu, Q. Huang, and L. Guo, "Luteolin inhibits proliferation and affects the function of stimulated rat synovial fibroblasts," Cell Biology International, vol. 33, no. 2, pp. 135-147, 2009.

[58] Y. Kawahito, M. Kondo, Y. Tsubouchi et al., "15-deoxy- $\Delta 12$, 14-PGJ2 induces synoviocyte apoptosis and suppresses adjuvant-induced arthritis in rats," Journal of Clinical Investigation, vol. 106, no. 2, pp. 189-197, 2000.

[59] X. Li, Y. Sun, J. Bao et al., "Functional role of PPAR- $\gamma$ on the proliferation and migration of fibroblast-like synoviocytes in rheumatoid arthritis," Scientific Reports, vol. 7, no. 1, Article ID 12671, 2017.

[60] X. Yang, Y. H. Li, R. L. Lv et al., "Study on the multitarget mechanism and key active ingredients of herba siegesbeckiae and volatile oil against rheumatoid arthritis based on network pharmacology," Evidence-Based Complementary and Alternative Medicine, vol. 2019, Article ID 8957245, 2019.

[61] R. Craig, A. Larkin, A. M. Mingo et al., "p38 MAPK and NF$\kappa \mathrm{B}$ collaborate to induce interleukin- 6 gene expression and release," Journal of Biological Chemistry, vol. 275, no. 31, pp. 23814-23824, 2000.

[62] L. Wu, X. Huang, L. Li, H. Huang, R. Xu, and W. Luyten, "Insights on biology and pathology of HIF- $1 \alpha /-2 \alpha$, TGF $\beta /$ BMP, wnt $/ \beta$-catenin, and NF- $\kappa$ B pathways in osteoarthritis," Current Pharmaceutical Design, vol. 18, no. 22, pp. 3293-3312, 2012. 